\title{
Editorial to the Special Issue "80 Years of Professor Wigner's Seminal Work: On Unitary Representations of the Inhomogeneous Lorentz Group"
}

\author{
Julio Marny Hoff da Silva
}

check for

updates

Citation: Hoff da Silva, J.M. Editorial to the Special Issue " 80 Years of Professor Wigner's Seminal Work: On Unitary Representations of the Inhomogeneous Lorentz Group". Universe 2021, 7, 310. https:/ / doi.org/10.3390/universe7080310

Received: 11 August 2021

Accepted: 13 August 2021

Published: 21 August 2021

Publisher's Note: MDPI stays neutral with regard to jurisdictional claims in published maps and institutional affiliations.

Copyright: (C) 2021 by the author. Licensee MDPI, Basel, Switzerland. This article is an open access article distributed under the terms and conditions of the Creative Commons Attribution (CC BY) license (https:/ / creativecommons.org/licenses/by/ $4.0 /)$.
Departamento de Física, Universidade Estadual Paulista, UNESP, Av. Dr. Ariberto Pereira da Cunha, 333 , Guaratinguetá 12516-410, Brazil; julio.hoff@unesp.br

The present Special Issue is dedicated to celebrate 80 years of the Professor Eugene Paul Wigner paper “On Unitary Representations of the Inhomogeneous Lorentz Group", published in 1939 [1]. It is almost impossible to have a fair measure of the impact of such a work. To name one of the most important achievements present in [1], the very concept of a particle is there constructed: a particle is an irreducible representation of the Poincarè group. For the contemporary reader, this assertion may sound as a kind of truism, but it certainly deserves some additional attention.

Poincarè transformations leave the Minkowski metric invariant. They may quite well be understood as symmetries of spacetime in the absence of gravity. The fulcrum in the Wigner analysis is, then, to represent the group of spacetime symmetries in the Hilbert space - the space of quantum physical states-in such a way that this representation acts also in a symmetric way, where in this last case a symmetry means a set of transformations preserving probabilities. There are many intricate problems in such an endeavor.

To begin with, it is important to recall that, although very well known, the Lorentz group is not simple. It indeed has a nontrivial topology and the task of genuine or projective representation is a problem to be addressed. Besides, continuity is also a concept generally accepted as valid, but rarely proved. These formal, but definitely important, aspects were worked out and developed in a precise manner by Wigner. Moreover, as the Lorentz group is non-compact, there is no unitary representation of finite dimension for that. Unitarity, however, is indeed a property of representations preserving norms for a connected group (as Wigner himself demonstrated in 1931 [2]).

The crucial problem related above was solved by Wigner with the help of the induced representation method, by means of which the Little Group is reached. As a typical achievement resulting from such an approach, by selecting one particle state four-momentum representative for a massive particle as $(m, \overrightarrow{0})$, the Little Group condition engenders a constraint, leading to the recognizing of $S O(3)$ as the Little Group, endowed, thus, with momentum angular algebra (even for a rest momentum as the chosen one). That is to say: the spin of a massive particle (an intrinsic angular momentum indeed) is a quantum label without which it would not be possible to represent all the spacetime symmetries in Quantum Mechanics. The particle properties are then bound to the spacetime symmetries. After 56 pages of a rigorous analysis, this is the kind of beauty arising from the Wigner analysis.

The contributions to this Special Issue may be separated out into Reviews and Research papers. In a broad-brush picture it may be reported as follows: in the paper [3] the effects of chirality in the spin entanglement between a lepton and a neutrino are investigated. The results point to an interesting framework for measuring chiral oscillations and, as a consequence, the intrinsic (bi)spinorial fermionic structure. The paper [4] deals in detail and carefully with the gauge invariance of the bosonic measure in the generating functional of chiral gauge theories, a point usually commented on, but not addressed. Going further in the Special Issue contributions, in the paper [5], the algebra of quaternions is used to achieve interesting results for the relativistic combination of non-collinear tri-velocities. As 
a result, the nontrivial angular dependence for such combinations is naturally absorbed in the quaternion multiplication, the same happening with other relevant results. In the paper [6], the outlines of a framework to discuss quantum gravity is presented. The ideas, comments and thoughts are clearly exposed, entailing a rich discussion. The resulting model argues that what we perceive as classical spacetime is actually the configuration space of its content.

Moving to the reviews part of the Special Issue, the review [7] brings a discussion about the special care devoted to the strongly continuous representation concept in the Wigner analysis. The relevant theorems and propositions related to the continuity concept in representation theory are reviewed in detail. The review [8] approaches the structure of gauge theories of gravity and post-Riemann geometries, discussing their recent developments and framing the analysis in the scope of understanding the very nature of spacetime and gravity in regimes where the General Relativity framework may come to debacle. The review [9] investigates the concept and properties of Dirac, Weyl and Majorana fermions in the four-dimensional Minkowski spacetime. Special attention is devoted to the quantum aspects of Weyl and Majorana fermions, discussing and clearing some misconceptions presented in the literature. The effective actions for these fermions coupled to gauge fields are reviewed in detail, and the relation between chiral and trace anomalies is extensively approached.

This Special Issue provides a well balanced mix of reviews and new results, culminating in an interesting volume celebrating and appreciating the Wigner work, but also studying physically relevant problems and methods in contemporary physics. There is rich material covering a wide range of areas, such as Quantum Mechanics and Quantum Field Theory, Mathematical Physics, and Gravitation. In some sense, the variety of subjects approached here is also a tribute to the robust foundational work of Professor Wigner: deep roots provide leafy and branched trees.

Funding: This work was supported by the Conselho Nacional de Desenvolvimento Científico CNPq (grant No. 303561/2018-1).

Institutional Review Board Statement: Not applicable.

Informed Consent Statement: Not applicable.

Data Availability Statement: Not applicable.

Acknowledgments: JMHS thanks to CNPq (grant No. 303561/2018-1) for financial support.

Conflicts of Interest: The author declares no conflict of interest.

\section{References}

1. Wigner, E.P. On Unitary Representations of the Inhomogeneous Lorentz Group. Annals Math. 1939, 40, 149. [CrossRef]

2. Wigner, E.P. Gruppentheorie und ihre Anwendungen auf die Quantenmechanik der Atomspektren; Springer: Braunschweig, Germany, 1931; p. 251.

3. Bittencourt, V.A.S.V.; Bernardini, A.E.; Blasone, M. Lepton-Antineutrino Entanglement and Chiral Oscillations. Universe 2021, 7, 293. [CrossRef]

4. de Lima e Silva, G.; Girardi, T.J.; Dias, S.A. On Gauge Invariance of the Bosonic Measure in Chiral Gauge Theories. Universe 2021, 7, 283. [CrossRef]

5. Berry, T.; Visser, M. Relativistic Combination of Non-Collinear 3-Velocities Using Quaternions. Universe 2020, 6, 237. [CrossRef]

6. Ziaeepour, H. Making a Quantum Universe: Symmetry and Gravity. Universe 2020, 6, 194. [CrossRef]

7. Hoff da Silva, J.M.; Caires da Rocha, G.M. Strongly Continuous Representations in the Hilbert Space: A Far-Reaching Concept. Universe 2021, 7, 285. [CrossRef]

8. Cabral, F.; Lobo, F.S.N.; Rubiera-Garcia, D. Fundamental Symmetries and Spacetime Geometries in Gauge Theories of Gravity-Prospects for Unified Field Theories. Universe 2020, 6, 238. [CrossRef]

9. Bonora, L.; Soldati, R.; Zalel, S. Dirac, Majorana, Weyl in 4D. Universe 2020, 6, 111. [CrossRef] 